\title{
Optimal Liquidation Behaviour Analysis for Stochastic Linear and Nonlinear Systems of Self-Exciting Model with Decay
}

\author{
Jiangming $\mathrm{Ma} \mathbb{D}^{1}$ and Xiankang Luo $\mathbb{D}^{2}$ \\ ${ }^{1}$ School of Economics, Xihua University, Chengdu, Sichuan 610039, China \\ ${ }^{2}$ Faculty of Science, Yibin University, Yibin, Sichuan 644000, China \\ Correspondence should be addressed to Xiankang Luo; xkluo1978@163.com
}

Received 21 July 2021; Revised 20 August 2021; Accepted 21 August 2021; Published 2 September 2021

Academic Editor: Yue Song

Copyright (c) 2021 Jiangming Ma and Xiankang Luo. This is an open access article distributed under the Creative Commons Attribution License, which permits unrestricted use, distribution, and reproduction in any medium, provided the original work is properly cited.

\begin{abstract}
When the market environment changes, we extend the self-exciting price impact model and further analysis of investors' liquidation behaviour. It is assumed that the model is accompanied by an exponential decay factor when the temporary impact and its coefficient are linear and nonlinear. Using the optimal control method, we obtain that the optimal liquidation behaviours satisfy the second-order nonlinear ODEs with variable coefficients in the case of linear and nonlinear temporary impact. Next, we solve the ODEs and get the form of the investors' optimal liquidation behaviour in four cases. Furthermore, we prove the decreasing properties of the optimal liquidation behaviour under the linear temporary impact. Through numerical simulation, we further explain the influence of the changed parameters $\rho, a, b, x$, and $\alpha$ on the investors' liquidation strategy $X_{t}$ in twelve scenarios. Some interesting properties have been found.
\end{abstract}

\section{Introduction}

Bertsimas and Lo [1] proposed the optimal execution model which has a linear and discrete price impact model in a fixed time. They got the optimal liquidation behaviours by an optimal control method in some cases. Two years later, Almgren and Chriss [2] used the minimization of meanvariance function to think the expected costs and risks. They obtained an optimal closed-form solution of static behaviour. When this model is proposed, it is quickly studied by a large number of scholars in different situations. Almgren [3] further extended the previous model which is assumed that the temporary impact is nonlinear and got the form of the optimal liquidation behaviour.

Huberman and Stanzl [4] studied the linear and nonlinear fixed impact and found that there exists no arbitrage phenomenon only when the market impact is linear. Gatheral and Schied [5] provided the behaviour of optimal expectation transaction cost which is satisfied with a HJB equation. In this paper, the optimal behaviours of two benchmark models are obtained, and it is found that the optimal behaviours of the two models are irrelevant unless there are extremely unrealistic situations and parameters. Gatheral et al. [6] researched the optimal trading strategy in which the temporary market impact has a decay factor. They proved some properties of the optimal investment liquidation behaviour and gave some specific form of the optimal behaviour under some special decay functions. Lehalle and Neuman [7] introduced Markovian signals under the optimal trading framework proposed by Gatheral et al. [6] and got the existence and uniqueness of optimal trading behaviour. Moreover, the authors gave the optimal investment behaviour of investors under the condition of Ornstein Uhlenbeck signal and temporary market impact with exponential decay. Based on the premise of no dynamic arbitrage, Gatheral [8] studied the optimal investment behaviour under the condition of market impact with decay factors. He gave the specific form of the optimal investment behaviour from the case of fixed and temporary market impact with different decay factors. Curato et al. [9] investigated the optimal execution of a large trade when the model has nonlinear transient impact and decay factors. 
They got the specific forms of the optimal strategy in some special cases. Barger and Lorig [10] investigated the optimal liquidation behaviours when the parameters of price impact are stochastic processes. They obtained the form of optimal liquidation behaviours by solving the HJB equation. Based on the Bayesian learning and dynamic programming techniques, Bismuth et al. [11] researched the optimal portfolio choice, portfolio liquidation, and portfolio transition problems. Through the optimal control method, they found that the optimal behaviour is the solution of the HJB equation. When the Almgren-Chriss model has $n$ riskaverse agents, Schied and Zhang [12] discussed the Nash equilibria and closed-form solutions of optimal liquidation by solving the ODE equation. Based on the Almgren-Chriss framework, Vaes and Hauser [13] considered a volume uncertainty model and proved that a risk-averse trader has benefit when he delays trades. Meanwhile, Ekren and Nadtochiy [14] researched the utility-based optimal hedging behaviour for a European-type option. From solving the solution of PDE, they obtained the optimal behaviours.

Based on Almgren-Chriss framework [2], a self-exciting price impact model is proposed by Caye and Muhle-Karbe [15]. They considered that a large sell order creates persistent selling pressure which not only incurs price impact but also increases the execution costs. However, they only discussed that the temporary impact is a linear function. When there exists a decay factor in the market, temporary impact and its coefficient are nonlinear functions, and the relevant problems have not been studied. Following the relevant literature, we add the exponential decay factor. Furthermore, when the temporary impact and its coefficient are linear and nonlinear, the optimal liquidation behaviours are obtained by the second-order nonlinear ODEs with variable coefficients.

The paper is organized as follows. In Section 2, we introduce the unaffected price process, the self-exciting price model, execution cost, and the objective function. In Section 3 , we present our main results: utilizing the optimal control method, we give the form of optimal liquidation behaviour when temporary impact and its coefficient are linear and nonlinear and discuss the properties of optimal liquidation behaviours. In Section 4, from seven different numerical examples, we discuss the effect of parameter changes on liquidation behaviours and show the corresponding financial interpretations. In Section 5, we conclude our research and give the future research direction.

\section{Statement of Background}

The following assumptions of the self-exciting price impact model are given. The trading time is fixed in $[0, T] . X_{t}$ represents the holdings at time $t$, where $X_{0}=x$ and $X_{T}=0$. Meanwhile, $X_{t}$ which is absolutely continuous and bounded with derivative $\dot{X}_{t}$ and $X_{t}=x+\int_{0}^{t} X_{t} \mathrm{~d} t$ satisfies $\int_{0}^{T}\left(\dot{X}_{t}\right)^{2} \mathrm{~d} t<\infty$.

The self-exciting price impact model contains unaffected price process as follows:

$$
S_{t}^{0}:=\sigma d W_{t},
$$

where $W_{t}$ is the standard Brownian motion. Thus, the specific form of the self-exciting price impact is assumed to be

$$
S_{t}:=S_{t}^{0}+\left(a+b\left(x-X_{t}\right)\right) \dot{X}_{t}, \quad t \in[0, T],
$$

where $a>0$ and $b>0$.

Therefore, the total execution cost of investors is

$$
\mathscr{C}(X):=x S_{0}+\int_{0}^{T} \dot{X}_{t} S_{t} \mathrm{~d} t .
$$

Only when the execution cost is the minimum, investors can get the maximum returns. So, the objective function of investors is

$$
\operatorname{minimize} \mathbb{E}[\mathscr{C}(X)] \text {. }
$$

\section{Main Results}

Caye and Muhle-Karbe [15] only discussed liquidation behaviours when the coefficient of temporary impact is linear. When there exists a decay factor in the market, the relevant literature has not been studied. In this paper, we introduce the classical exponential decay factor in the selfexciting price impact model. Next, we study the optimal liquidation behaviour of investors when the market has exponential decay under the four cases.

Theorem 1. When there is equation (2) with exponential decay $e^{-\rho t}$, the mean optimization strategy is the unique solution of the following nonlinear ODE with variable coefficients:

$$
2\left[a+b\left(x-X_{t}\right)\right] \ddot{X}_{t}-b \dot{X}_{t}^{2}-2 \rho\left(a+b\left(x-X_{t}\right)\right) \dot{X}_{t}=0,
$$

with boundary conditions as follows:

$$
\begin{aligned}
& X_{0}=x, \\
& X_{T}=0 .
\end{aligned}
$$

The solution of equation (5) is

$$
X_{t}=\frac{a}{b}+x-\frac{C_{1}}{b} e^{t} e^{\rho \nu}\left[C_{2}+(1 / 2) \int_{0}^{t} e^{\rho v} \mathrm{~d} v\right]^{-1} \mathrm{~d} v
$$

Proof. When the market has an exponential decay, equation (2) is

$$
S_{t}:=S_{t}^{0}+e^{-\rho t}\left(a+b\left(x-X_{t}\right)\right) \dot{X}_{t}, \quad t \in[0, T] .
$$

From equations (3) and (8), we get 


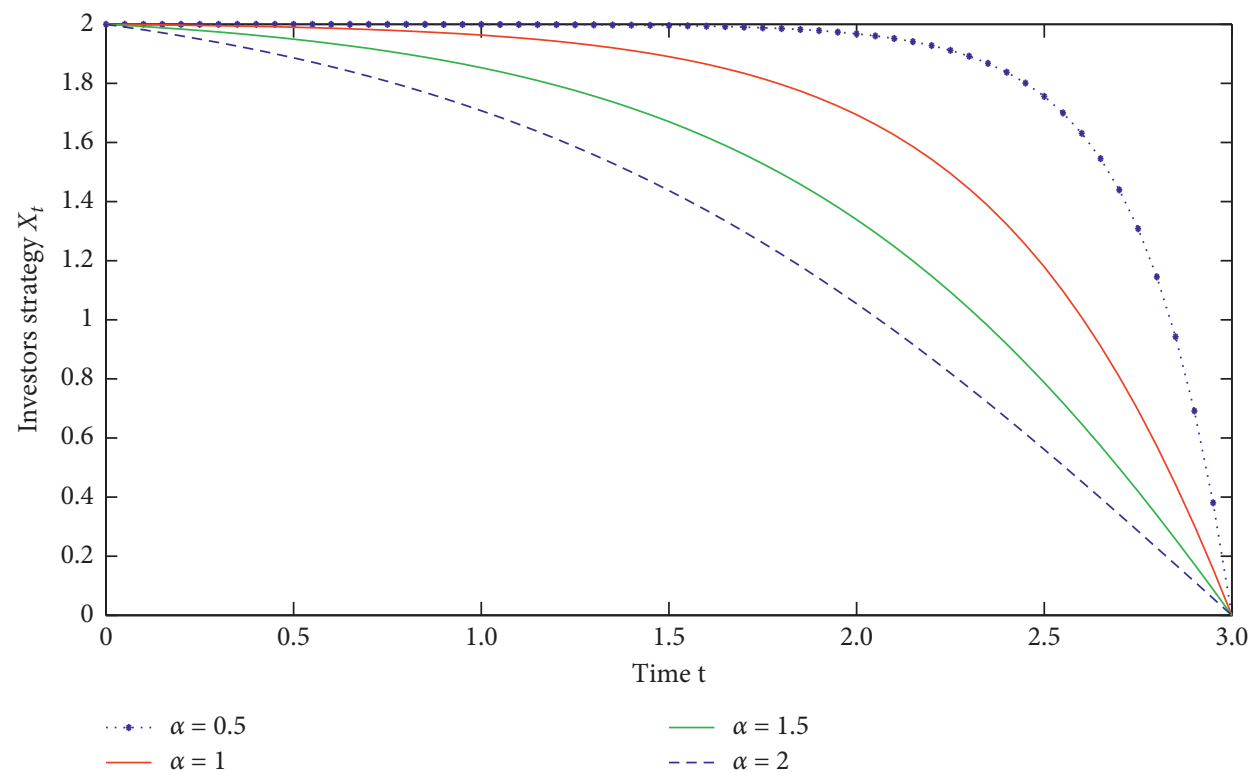

Figure 1: Influence of $\alpha$ change on equation (26) when $\rho=2, a=0.6, b=0.5$, and $x=2$.

$$
\begin{aligned}
\mathscr{C}(X):= & x S_{0}+\int_{0}^{T} \dot{X}_{t} S_{t} \mathrm{~d} t=x S_{0} \\
& +\int_{0}^{T} \dot{X}_{t}\left(S_{t}^{0}+e^{-\rho t}\left(a+b\left(x-X_{t}\right)\right) \dot{X}_{t}\right) \mathrm{d} t \\
= & \int_{0}^{T} \sigma X_{t} \mathrm{~d} W_{t}+\int_{0}^{T} e^{-\rho t}\left(a+b\left(x-X_{t}\right)\right) \dot{X}_{t}{ }^{2} \mathrm{~d} t .
\end{aligned}
$$

Combined with the properties of Ito integral and equation (4), the above equation is changed to be

$$
\operatorname{minimize} \mathbb{E}[\mathscr{C}(X)]=\int_{0}^{T} e^{-\rho t}\left(a+b\left(x-X_{t}\right)\right) X_{t}^{2} \mathrm{~d} t .
$$

In order to get the solution of equation (10), we use the Euler-Lagrange equation to get the following second-order nonlinear ODE with variable coefficients:

$$
2\left[a+b\left(x-X_{t}\right)\right] \ddot{X}_{t}-b \dot{X}_{t}^{2}-2 \rho\left(a+b\left(x-X_{t}\right)\right) \dot{X}_{t}=0 .
$$

Let $Y_{t}=a+b\left(x-X_{t}\right)$, equation (11) is changed as follows:

$$
2 Y_{t} \ddot{Y}_{t}-\dot{Y}_{t}^{2}-2 \rho Y_{t} \dot{Y}_{t}=0 .
$$

From the [16], the solution of equation (12) is

$$
Y_{t}=C_{1} e_{0}^{t} e^{\rho v}\left[C_{2}+(1 / 2) \int_{0}^{t} e^{\rho v} \mathrm{~d} v\right]^{-1} \mathrm{~d} v
$$

Thus, the solution of equation (5) is

$$
X_{t}=\frac{a}{b}+x-\frac{C_{1}}{b} e^{\int_{0}^{t} e^{\rho v}\left[C_{2}+(1 / 2) \int_{0}^{t} e^{\rho v} \mathrm{~d} \nu\right]^{-1} \mathrm{~d} \nu} \text {. }
$$

Remark 1. In Theorem 1, we address the optimal liquidation behaviour when there is a self-exciting model with exponential decay and give the specific form of optimal liquidation behaviour. That is, the coefficient of temporary impact is multiplied by the exponential function. In fact, when $\rho=0$, our model is reduced to the price model of Caye and Muhle-Karbe [15].

Theorem 2. Under the assumption of Theorem 1, the optimal strategy $X_{t}$ from equation (14) is deterministic, absolutely continuous, and decreasing.

Proof

$$
\begin{aligned}
\mathscr{C}(X) & =\int_{0}^{T} e^{-\rho t}\left(a+b\left(x-X_{t}\right)\right) X_{t}^{2} \mathrm{~d} t \\
& =(a+b x) \int_{0}^{T} e^{-\rho t} X_{t}^{2} \mathrm{~d} t-b \int_{0}^{T} e^{-\rho t} X_{t} X_{t}^{2} \mathrm{~d} t \\
& =\mathscr{F}(X) .
\end{aligned}
$$

Let $Y=X-X^{*}$, then we get 


$$
\begin{aligned}
& \mathscr{F}(X)=\mathscr{F}\left(Y+X^{*}\right) \\
& =(a+b x) \int_{0}^{T} e^{-\rho t}\left(\dot{Y}_{t}+\dot{X}_{t}^{*}\right)^{2} \mathrm{~d} t-b \int_{0}^{T} e^{-\rho t}\left(Y_{t}+X^{*}\right)\left(\dot{Y}_{t}+\dot{X}_{t}^{*}\right)^{2} \mathrm{~d} t \\
& =(a+b x) \int_{0}^{T} e^{-\rho t}\left(\dot{Y}_{t}{ }^{2}+2 \dot{Y}_{t} \dot{X}_{t}^{*}+\left(\dot{X}_{t}^{*}\right)^{2}\right) \mathrm{d} t-b \int_{0}^{T} e^{-\rho t}\left(Y_{t}+X^{*}\right)\left(\dot{Y}_{t}{ }^{2}+2 \dot{Y}_{t} \dot{X}_{t}^{*}+\left(\dot{X}_{t}^{*}\right)^{2}\right) \mathrm{d} t \\
& =(a+b x) \int_{0}^{T} e^{-\rho t}\left(\dot{Y}_{t}^{2}+2 \dot{Y}_{t} \dot{X}_{t}^{*}+\left(\dot{X}_{t}^{*}\right)^{2}\right) \mathrm{d} t \\
& -b \int_{0}^{T} e^{-\rho t}\left(Y_{t} \dot{Y}_{t}^{2}+2 Y_{t} \dot{Y}_{t} \dot{X}_{t}^{*}+Y\left(\dot{X}_{t}^{*}\right)^{2}+X_{t}^{*} \dot{Y}_{t}{ }^{2}+2 X_{t}^{*} \dot{Y}_{t} \dot{X}_{t}^{*}+X_{t}^{*}\left(\dot{X}_{t}^{*}\right)^{2}\right) \mathrm{d} t \\
& =\int_{0}^{T} e^{-\rho t}\left(a+b x-X_{t}^{*}\right)\left(\dot{X}_{t}^{*}\right)^{2} \mathrm{~d} t+\int_{0}^{T} e^{-\rho t}\left(a+b x-b X_{t}^{*}-b Y_{t}\right) \dot{Y}_{t}{ }^{2} \mathrm{~d} t \\
& +\int_{0}^{T} e^{-\rho t}\left[2(a+b x) \dot{Y}_{t} \dot{X}_{t}^{*}-2 b X_{t}^{*} \dot{Y}_{t} \dot{X}_{t}^{*}-b Y_{t}\left(\dot{X}_{t}^{*}\right)^{2}-2 b Y_{t} \dot{Y}_{t} \dot{X}_{t}^{*}\right] \mathrm{d} t \\
& =\int_{0}^{T} e^{-\rho t}\left(a+b x-X_{t}^{*}\right)\left(\dot{X}_{t}^{*}\right)^{2} \mathrm{~d} t+\int_{0}^{T} e^{-\rho t}\left(a+b x-b X_{t}^{*}-b Y_{t}\right) \dot{Y}_{t}{ }^{2} \mathrm{~d} t \\
& +\int_{0}^{T} e^{-\rho t} Y_{t}\left[\begin{array}{c}
2 \rho(a+b x) \dot{X}_{t}^{*}-2(a+b x) X_{t}^{*}-2 b \rho X_{t}^{*} \dot{X}_{t}^{*} \\
+2 b\left(\dot{X_{t}^{*}}\right)^{2}+2 b X_{t}^{*} \ddot{X}_{t}^{*}-b\left(\dot{X}_{t}^{*}\right)^{2}
\end{array}\right] \mathrm{d} t \\
& -2 b \int_{0}^{T} e^{-\rho t} Y_{t} \dot{Y}_{t} \dot{X}_{t}^{*} \mathrm{~d} t=\int_{0}^{T} e^{-\rho t}\left(a+b x-X_{t}^{*}\right)\left(\dot{X}_{t}^{*}\right)^{2} \mathrm{~d} t \\
& +\int_{0}^{T} e^{-\rho t}\left(a+b x-b X_{t}^{*}-b Y_{t}\right) \dot{Y}_{t}^{2} \mathrm{~d} t \\
& -\int_{0}^{T} e^{-\rho t} Y_{t}\left[2\left(a+b\left(x-X_{t}^{*}\right)\right) \ddot{X}_{t}^{*}-2 \rho\left(a+b\left(x-X_{t}^{*}\right)\right) \dot{X}_{t}^{*}-b\left(\dot{X}_{t}^{*}\right)^{2}\right] \mathrm{d} t \\
& -2 b \rho \int_{0}^{T} e^{-\rho t} Y_{t} X_{t}^{*} \dot{X}_{t}^{*} \mathrm{~d} t+2 b \int_{0}^{T} e^{-\rho t} Y_{t}\left(\dot{X}_{t}^{*}\right)^{2} \mathrm{~d} t+2 b \int_{0}^{T} e^{-\rho t} Y_{t} X_{t}^{*} \ddot{X}_{t}^{*} \mathrm{~d} t \\
& =\int_{0}^{T} e^{-\rho t}\left(a+b x-X_{t}^{*}\right)\left(\dot{X}_{t}^{*}\right)^{2} \mathrm{~d} t+\int_{0}^{T} e^{-\rho t}\left(a+b x-b X_{t}^{*}-b Y_{t}\right) \dot{Y}_{t}^{2} \mathrm{~d} t \\
& -b \rho \int_{0}^{T} e^{-\rho t} Y_{t}^{2} X_{t}^{*} X_{t}^{*} \mathrm{~d} t+b \int_{0}^{T} e^{-\rho t} Y_{t}^{2}\left(\dot{X}_{t}^{*}\right)^{2} \mathrm{~d} t+b \int_{0}^{T} e^{-\rho t} Y_{t}^{2} \ddot{X}_{t}^{*} \mathrm{~d} t \geq \mathscr{F}\left(X^{*}\right) .
\end{aligned}
$$

According to the basic assumptions of the model, combined with the following numerical simulation, we find that $\dot{X}_{t}$ satisfies $\dot{X}_{t}<0$. The sixth step is to get through integration by parts and $Y_{0}=Y_{T}=0$. Because $X_{t}^{*}$ satisfies equation (5), the third part of seventh step is removed. Using $x-X_{t}^{*}-Y_{t}=x-X_{t} \geq 0$, the third part of the seventh step is positive. We obtain the eighth step from integration by parts. Through equation (14) and numerical simulation, $X_{t}^{*} \geqslant 0$ is verified. Thus, $X_{t}$ is decreasing.

Remark 2. In Theorem 2, we find that the optimal liquidation behaviour still has the same properties as the liquidation behaviour without decay in Caye and Muhle-Karbe [15] when the market has decayed.

In the last part, we discuss the optimal liquidation behaviour of investors when the temporary market impact is a linear function. Meanwhile, Almgren [3], Gatheral [8],
Curato et al. [9], and Horst and Naujokat [17] studied the optimal liquidation behaviour of investors when the temporary impact is nonlinear in different cases. When the market is accompanied by a decay factor and the temporary market impact is nonlinear, the optimal liquidation behaviour of investors is not studied by Caye and Muhle-Karbe [15]. Through the study of the existing literature, we assume that the temporary impact is a power function. Thus, when market has an exponential decay, the new price impact model is

$$
S_{t}:=S_{t}^{0}+e^{-\rho t}\left(a+b\left(x-X_{t}\right)\right)\left(\dot{X}_{t}\right)^{\alpha}, \quad t \in[0, T]
$$

Theorem 3. When there is equation (17) with exponential decay $e^{-\rho t}$, the mean optimization behaviour is the unique 


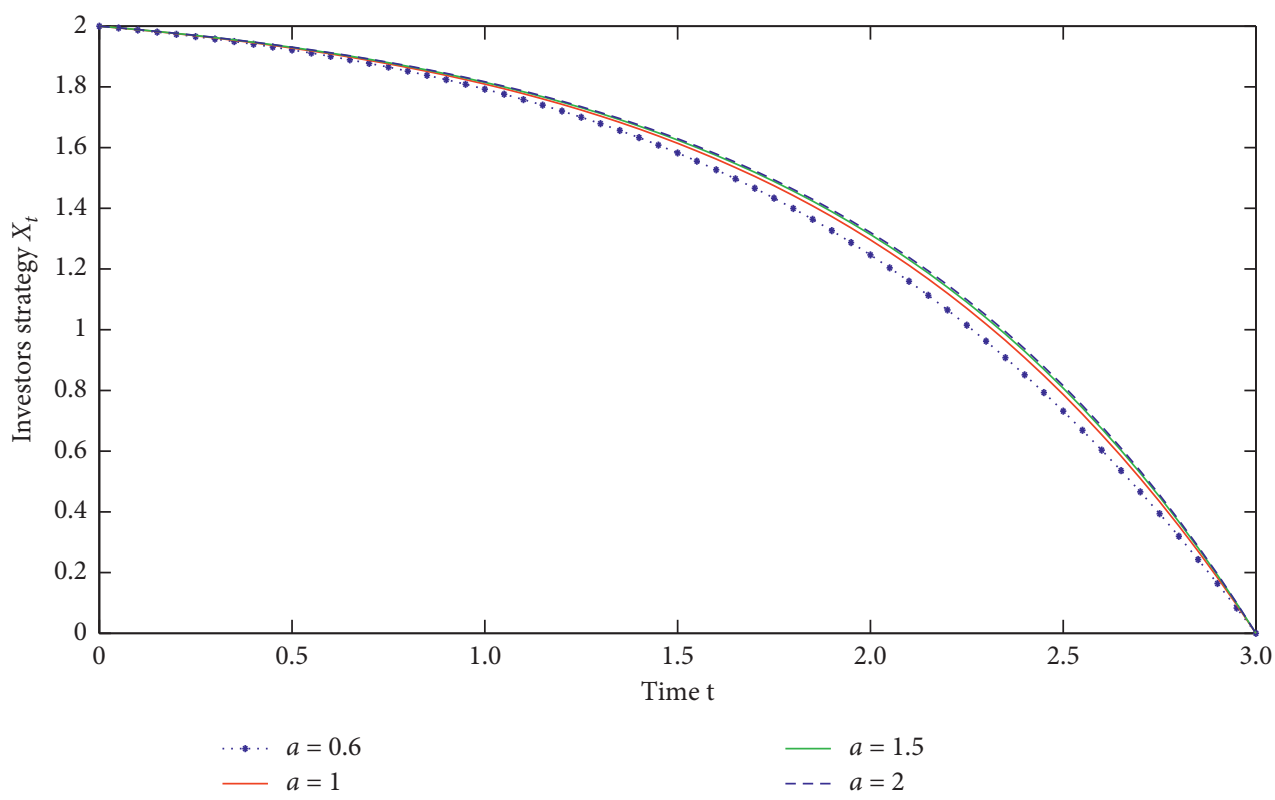

Figure 2: Influence of $a$ change on equation (14) when $\alpha=1, \rho=1, b=0.5$, and $x=2$.

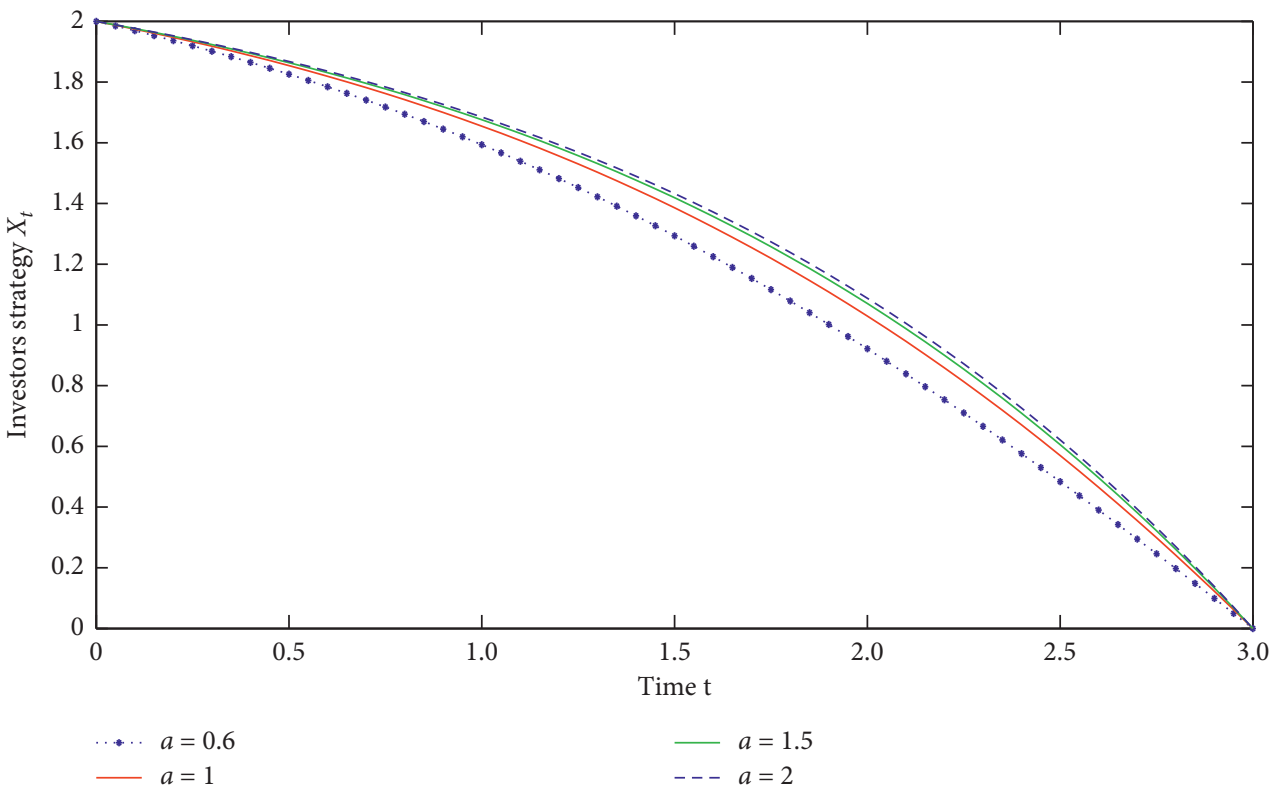

Figure 3: Influence of $a$ change on equation (26) when $\alpha=1.5, \rho=1, b=0.5$, and $x=2$.

solution of the following nonlinear ODE with variable coefficients:

$$
\begin{gathered}
\alpha(\alpha+1)\left[a+b\left(x-X_{t}\right)\right] \ddot{X}_{t}-b \alpha \dot{X}_{t}^{2} \\
-\rho(\alpha+1)\left(a+b\left(x-X_{t}\right)\right) \dot{X}_{t}=0,
\end{gathered}
$$

with boundary conditions as follows:

$$
\begin{aligned}
& X_{0}=x, \\
& X_{T}=0 .
\end{aligned}
$$

The solution of equation (18) is $X_{t}=\frac{a}{b}+x-\frac{C_{1}}{b} e^{t} e^{(\rho / \alpha) v}\left[C_{2}+(\alpha / \alpha+1) \int_{0}^{t} e^{(\rho / \alpha) v} \mathrm{~d} v\right]^{-1} \mathrm{~d} v$. 


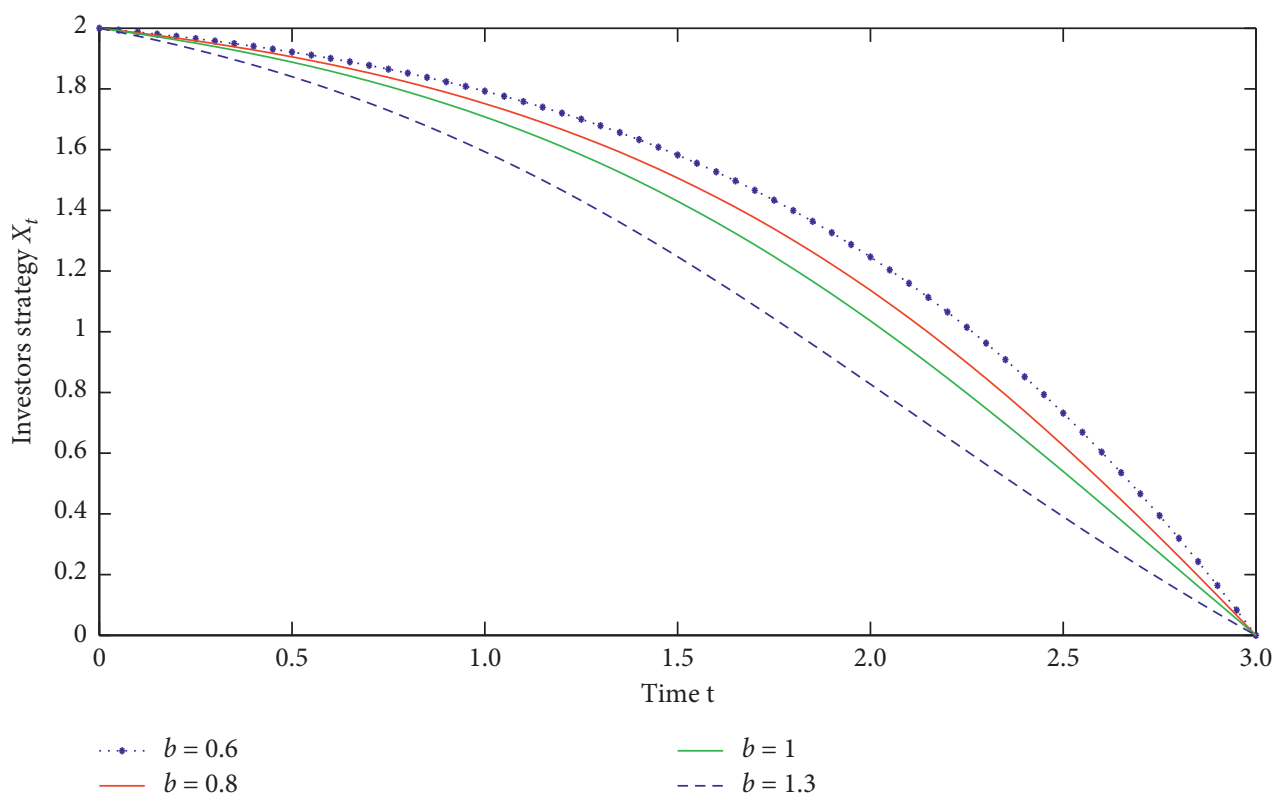

FIGURE 4: Influence of $b$ change on equation (14) when $\alpha=1, \rho=1, a=0.6$, and $x=2$.

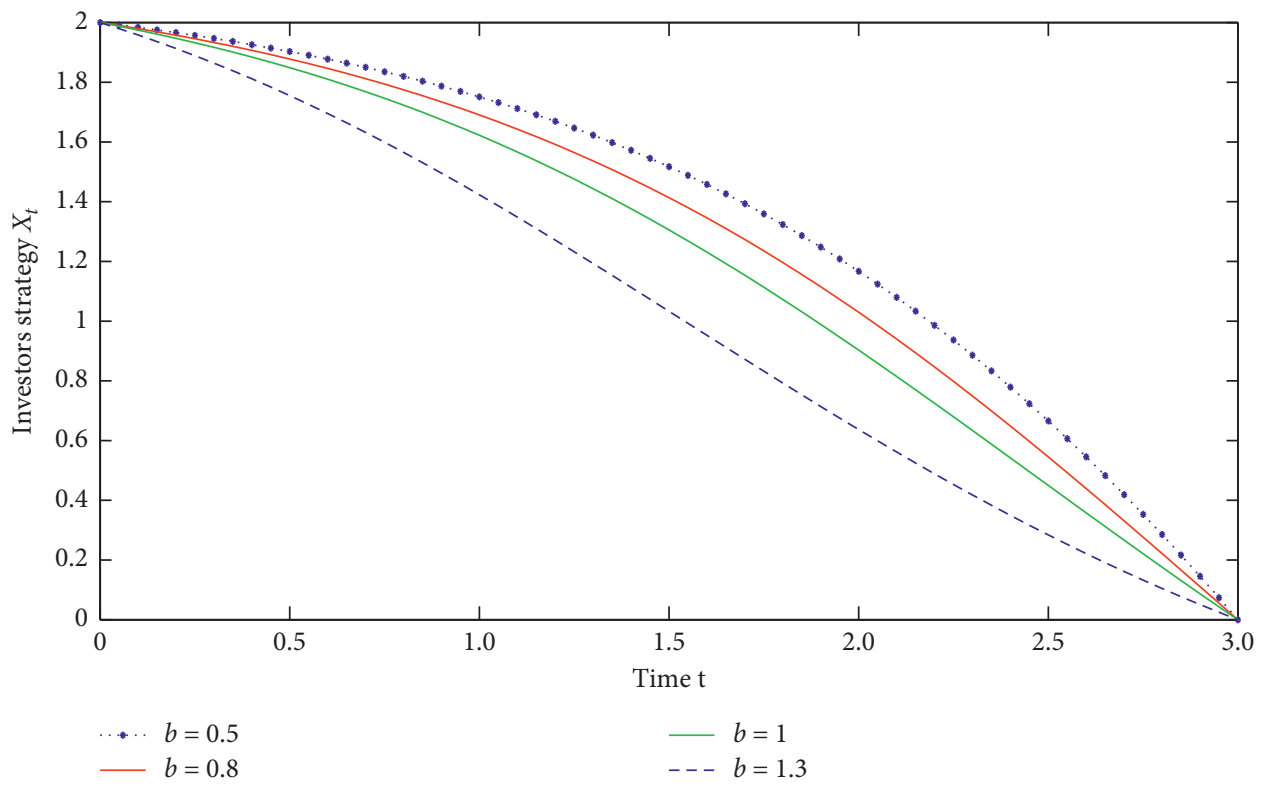

FIGURE 5: Influence of $b$ change on equation (26) when $\alpha=1.1, \rho=1, a=0.6$, and $x=2$.

Proof. From equations (3) and (17), we get

$$
\begin{aligned}
\mathscr{C}(X):= & x S_{0}+\int_{0}^{T} \dot{X}_{t} S_{t} \mathrm{~d} t=x S_{0} \\
& +\int_{0}^{T} \dot{X}_{t}\left(S_{t}^{0}+e^{-\rho t}\left(a+b\left(x-X_{t}\right)\right)\left(\dot{X}_{t}\right)^{\alpha}\right) \mathrm{d} t \\
= & \int_{0}^{T} \sigma X_{t} \mathrm{~d} W_{t}+\int_{0}^{T} e^{-\rho t}\left(a+b\left(x-X_{t}\right)\right) \dot{X}_{t}^{\alpha+1} \mathrm{~d} t .
\end{aligned}
$$

According to properties of Ito integral and equation (4), we get

$$
\operatorname{minimize} \mathbb{E}[\mathscr{C}(X)]=\int_{0}^{T} e^{-\rho t}\left(a+b\left(x-X_{t}\right)\right) \dot{X}_{t}{ }^{\alpha+1} \mathrm{~d} t
$$

Using the Euler-Lagrange equation, the following second-order nonlinear ODE is obtained with variable coefficients:

$$
\begin{gathered}
\alpha(\alpha+1)\left[a+b\left(x-X_{t}\right)\right] \ddot{X}_{t}-b \alpha \dot{X}_{t}^{2} \\
-\rho(\alpha+1)\left(a+b\left(x-X_{t}\right)\right) \dot{X}_{t}=0 .
\end{gathered}
$$




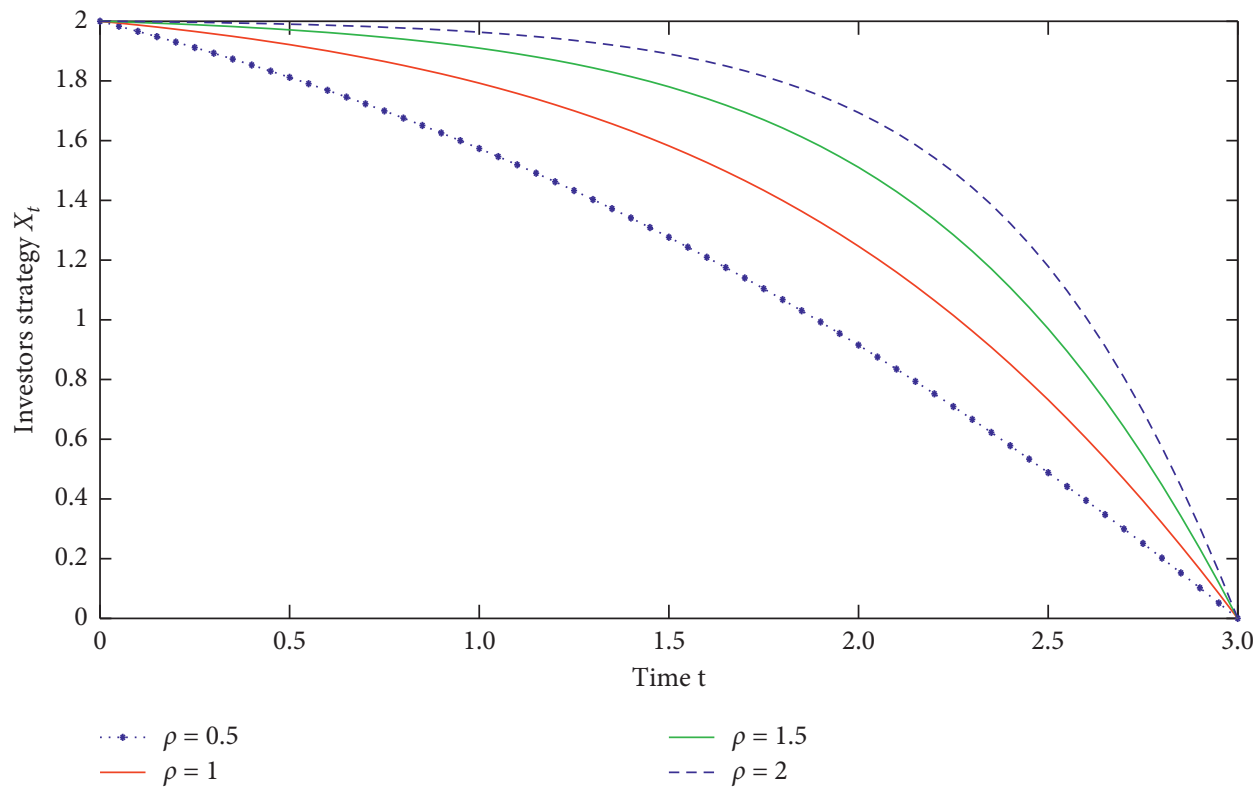

FIGURE 6: Influence of $\rho$ change on equation (14) when $\alpha=1, a=0.6, b=0.5$, and $x=2$.

Let $Y_{t}=a+b\left(x-X_{t}\right)$, equation (23) is changed as follows:

$$
\alpha(\alpha+1) Y_{t} \ddot{Y}_{t}-\alpha \dot{Y}_{t}^{2}-\rho(\alpha+1) Y_{t} \dot{Y}_{t}=0 .
$$

From [16], the solution of equation (24) is

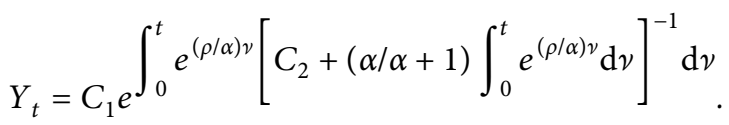

Thus, the solution of equation (18) is

$$
X_{t}=\frac{a}{b}+x-\frac{C_{1}}{b} e_{0}^{t} e^{(\rho / \alpha) v}\left[C_{2}+(\alpha / \alpha+1) \int_{0}^{t} e^{(\rho / \alpha) v} \mathrm{~d} v\right]^{-1} \mathrm{~d} v
$$

From equations (12) and (18), we find that equation (12) is the special form of equation (18) when $\alpha=1$.

Remark 3. In Theorem 3, we research the optimal liquidation behaviour when the market has exponential decay and temporary market impact is a nonlinear function. We can easily find that the price impact model of Caye and Muhle-Karbe [15] is our special form in this case of $\alpha=0$ and $\rho=0$.

Next, we investigate the liquidation behaviour under the coefficient of temporary impact as $e^{a+b\left(x-X_{t}\right)}$. Thus, equation (4) is changed to be

$$
S_{t}:=S_{t}^{0}+e^{a+b\left(x-X_{t}\right)} \dot{X}_{t}, \quad t \in[0, T]
$$

Theorem 4. When there is equation (27) with exponential decay $e^{-\rho t}$, the mean optimization behaviour is the unique solution of the following nonlinear ODE with variable coefficients:

$$
2 \ddot{X}_{t}-2 \rho \dot{X}_{t}-b \dot{X}_{t}^{2}=0
$$

with boundary conditions as follows:

$$
\begin{aligned}
& X_{0}=x, \\
& X_{T}=0 .
\end{aligned}
$$

The solution of equation (28) is

$$
\int e^{-(b / 2) X_{t}} \mathrm{~d} X_{t}=C_{1} e^{\rho t}+C_{2} \text {. }
$$

Proof. From equations (3) and (27), we get

$$
\begin{aligned}
\mathscr{C}(X) & :=x S_{0}+\int_{0}^{T} \dot{X}_{t} S_{t} \mathrm{~d} t \\
& =x S_{0}+\int_{0}^{T} e^{a+b\left(x-X_{t}\right)} e^{-\rho t} \dot{X}_{t}{ }^{2} \mathrm{~d} t \\
& =\int_{0}^{T} \sigma X_{t} \mathrm{~d} W_{t}+\int_{0}^{T} e^{a+b\left(x-X_{t}\right)-\rho t} \dot{X}_{t}{ }^{2} \mathrm{~d} t .
\end{aligned}
$$

From the properties of Ito integral and equation (4), we have

$$
\operatorname{minimize} \mathbb{E}[\mathscr{C}(X)]=\int_{0}^{T} e^{a+b\left(x-X_{t}\right)-\rho t} X_{t}^{2} \mathrm{~d} t .
$$

Using the Euler-Lagrange equation, the following nonlinear ODE with variable coefficients is obtained:

$$
2 \ddot{X}_{t}-2 \rho \dot{X}_{t}-b \dot{X}_{t}^{2}=0
$$

From [16], the solution of equation (33) is 


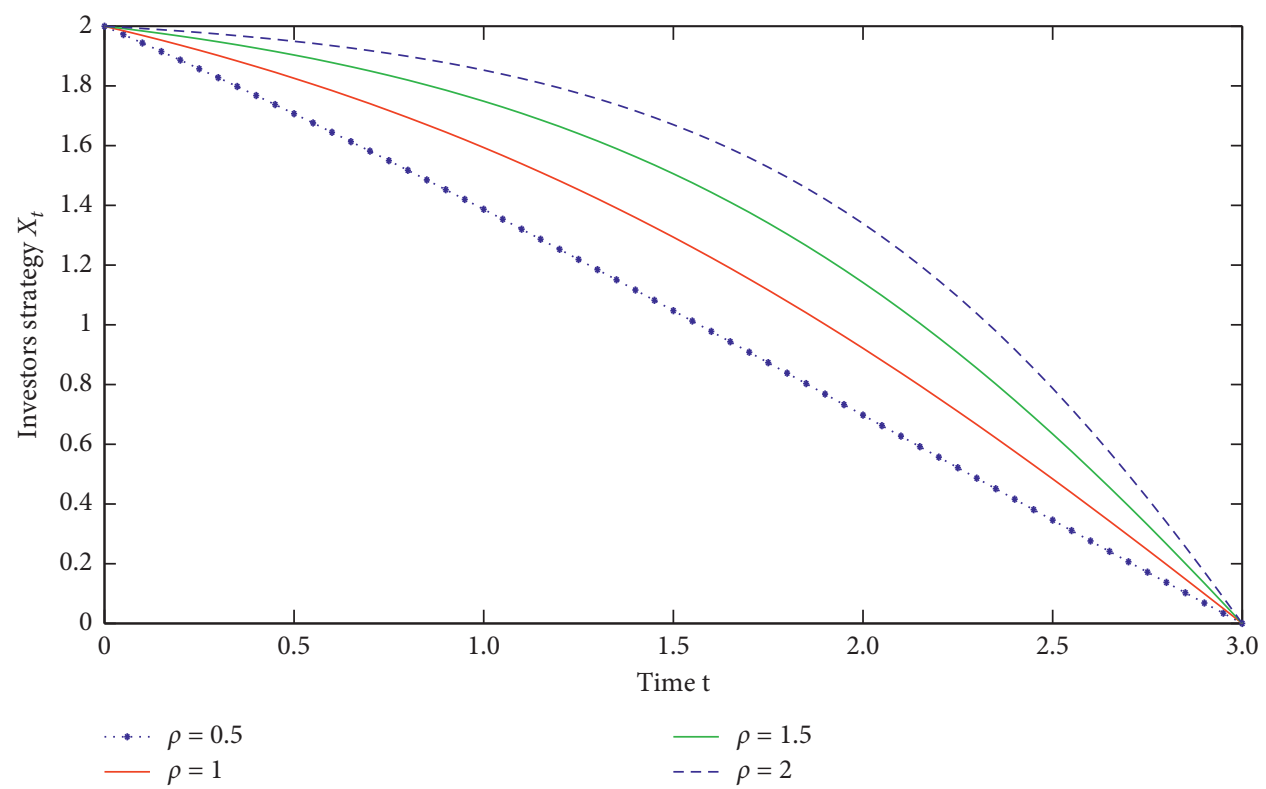

FIGURE 7: Influence of $\rho$ change on equation (26) when $\alpha=1.5, a=0.6, b=0.5$, and $x=2$.

$$
\int e^{-(b / 2) X_{t}} \mathrm{~d} X_{t}=C_{1} e^{\rho t}+C_{2}
$$

Remark 4. In Theorem 4, we research the optimal liquidation behaviour when coefficient of temporary impact is a nonlinear function and temporary impact has exponential decay based on Caye and Muhle-Karbe [15].

Theorem 5. Under the assumption of Theorem 4, the optimal behaviour $X_{t}$ from equation (34) is deterministic, absolutely continuous, and decreasing.
Proof

$$
\begin{aligned}
\mathscr{C}(X) & =\int_{0}^{T} e^{a+b\left(x-X_{t}\right)} e^{-\rho t} X_{t}^{2} \mathrm{~d} t \\
& =(a+b x) \int_{0}^{T} e^{-b X_{t}-\rho t} X_{t}^{2} \mathrm{~d} t=\mathscr{F}(X) .
\end{aligned}
$$

Let $Y=X-X^{*}$, then we get

$$
\begin{aligned}
\mathscr{F}(X)= & \mathscr{F}\left(Y+X^{*}\right) \\
= & e^{(a+b x)} \int_{0}^{T} e^{\left(-b X_{t}^{*}-b Y_{t}\right)} e^{-\rho t}\left(\dot{X}_{t}^{*}+\dot{Y}_{t}\right)^{2} \mathrm{~d} t \\
= & e^{(a+b x)} \int_{0}^{T} e^{\left(-b X_{t}^{*}-b Y_{t}\right)} e^{-\rho t}\left(\dot{X}_{t}^{*}+2 \dot{X}_{t}^{*} \dot{Y}_{t}+\dot{Y}_{t}{ }^{2}\right) \mathrm{d} t \\
= & e^{(a+b x)} \int_{0}^{T} e^{\left(-b X_{t}^{*}-b Y_{t}\right)} e^{-\rho t} \dot{X}_{t}^{*} \mathrm{~d} t+(a+b x) \int_{0}^{T} e^{\left(-b X_{t}^{*}-b Y_{t}\right)} e^{-\rho t}\left(2 \dot{X}_{t}^{*} \dot{Y}_{t}\right) \mathrm{d} t \\
& +e^{(a+b x)} \int_{0}^{T} e^{\left(-b X_{t}^{*}-b Y_{t}\right)} e^{-\rho t} \dot{Y}_{t}{ }^{2} \mathrm{~d} t \ldots{ }^{(a+b x)} \int_{0}^{T} e^{\left(-b X_{t}^{*}-b Y_{t}\right)} e^{-\rho t} \dot{X}_{t}^{*}{ }^{3} \mathrm{~d} t \\
\geqslant & (a+b x) \int_{0}^{T} e^{-\rho t} e^{-b X_{t}^{*} \dot{X}_{t}^{*}{ }^{3} \mathrm{~d} t .}
\end{aligned}
$$

From the properties of $\dot{X}_{t}, \dot{X}_{t}<0$, and integral, proof of Theorem 5 is easily obtained.

Remark 5. In Theorem 5, we research a property of the optimal liquidation behaviour when the market has exponential decay and the coefficients of temporary impact are nonlinear functions.

When the temporary impact is a power function, equation (27) is changed to be

$$
S_{t}:=S_{t}^{0}+e^{a+b\left(x-X_{t}\right)} \dot{X}_{t}{ }^{\alpha}, \quad t \in[0, T] .
$$




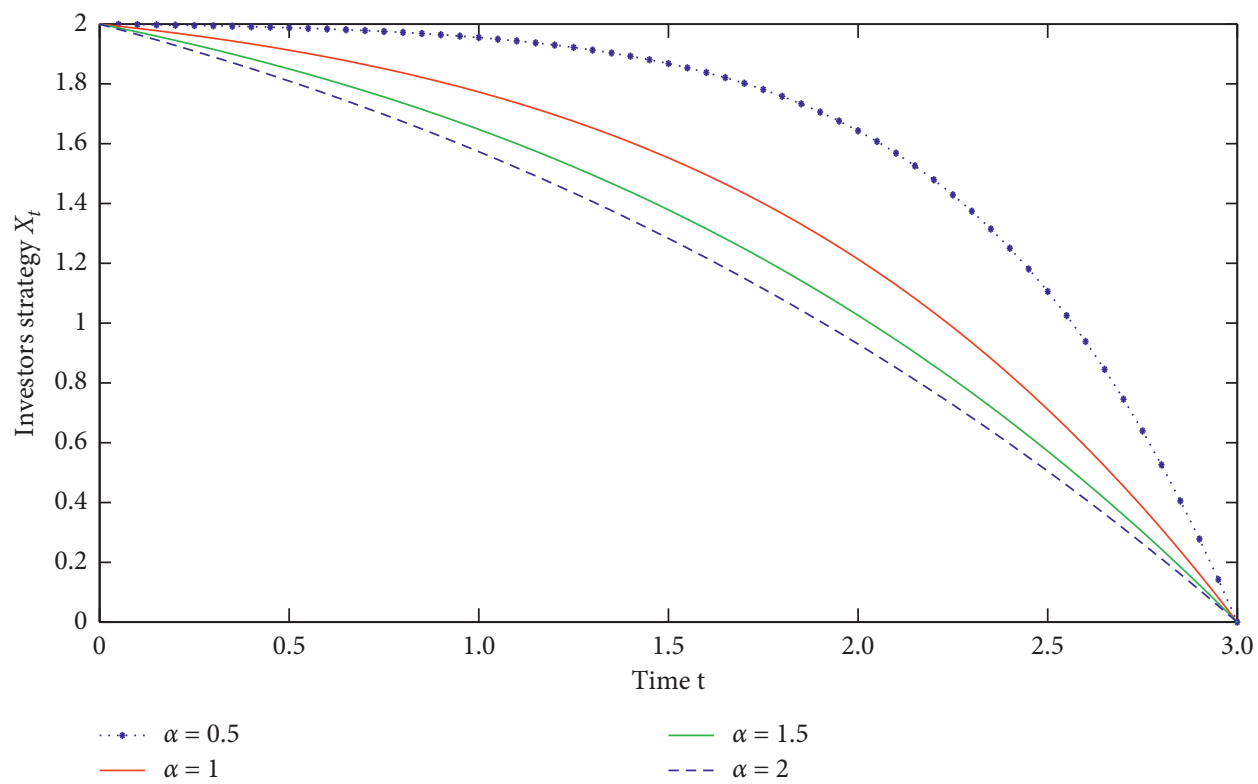

Figure 8: Influence of $\alpha$ change on equation (44) when $\rho=1, b=0.5$, and $x=2$.

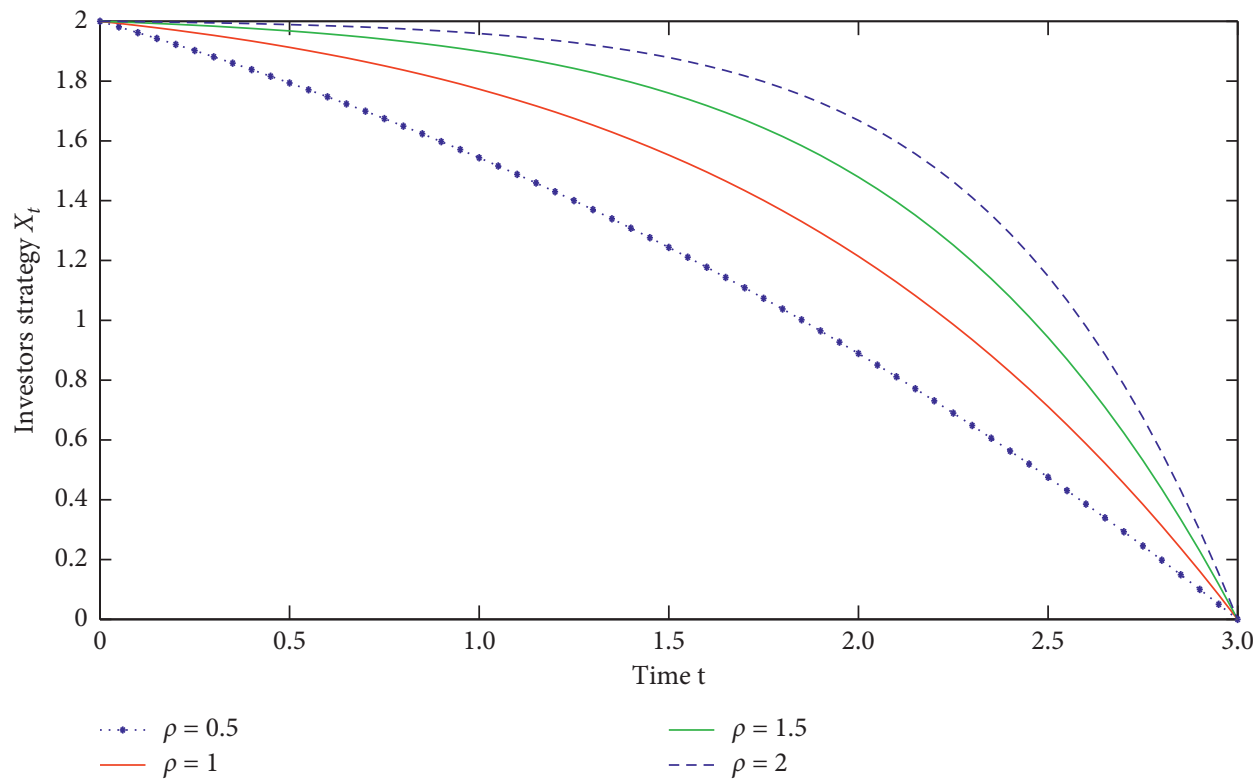

Figure 9: Influence of $\rho$ change on equation (34) when $\alpha=1, b=0.5$, and $x=2$.

Theorem 6. When there is equation (37) with exponential decay $e^{-\rho t}$, the mean optimization behaviour is the unique solution of the following nonlinear ODE with variable coefficients:

$$
\alpha(\alpha+1) \ddot{X}_{t}-\rho(\alpha+1) \dot{X}_{t}-b \alpha \dot{X}_{t}^{2}=0,
$$

with two-point boundary conditions as follows:

$$
\begin{aligned}
& X_{0}=x, \\
& X_{T}=0 .
\end{aligned}
$$

The solution of equation (38) is

$$
\int e^{-(b / \alpha+1) X_{t}} \mathrm{~d} X_{t}=C_{1} e^{(\rho / \alpha) t}+C_{2} .
$$

Proof. From equations (3) and (37), we get

$$
\begin{aligned}
\mathscr{C}(X) & :=x S_{0}+\int_{0}^{T} \dot{X}_{t} S_{t} \mathrm{~d} t \\
& =x S_{0}+\int_{0}^{T} e^{a+b\left(x-X_{t}\right)} e^{-\rho t}\left(\dot{X}_{t}\right)^{\alpha+1} \mathrm{~d} t \\
& =\int_{0}^{T} \sigma X_{t} \mathrm{~d} W_{t}+\int_{0}^{T} e^{a+b\left(x-X_{t}\right)-\rho t} \dot{X}_{t}^{\alpha+1} \mathrm{~d} t .
\end{aligned}
$$




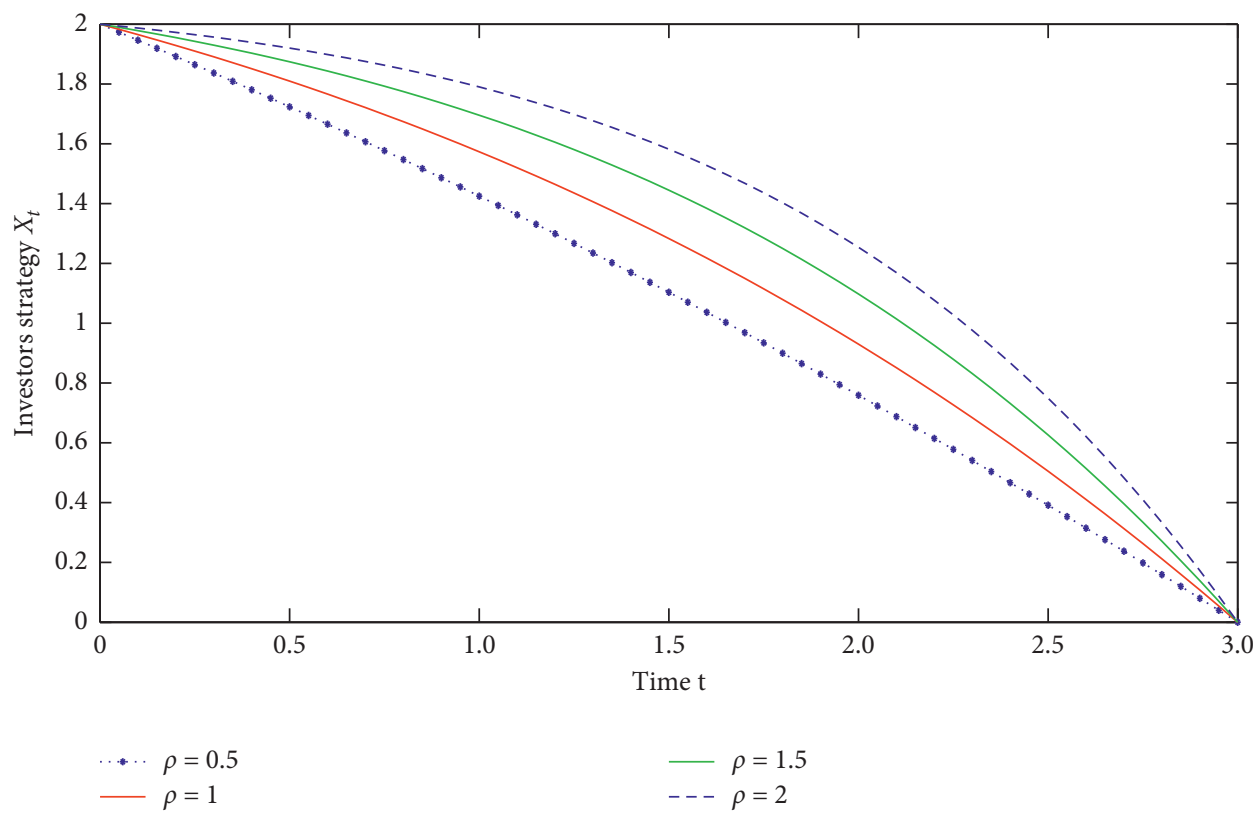

Figure 10: Influence of $\rho$ change on equation (44) when $\alpha=2, b=0.5$, and $x=2$.

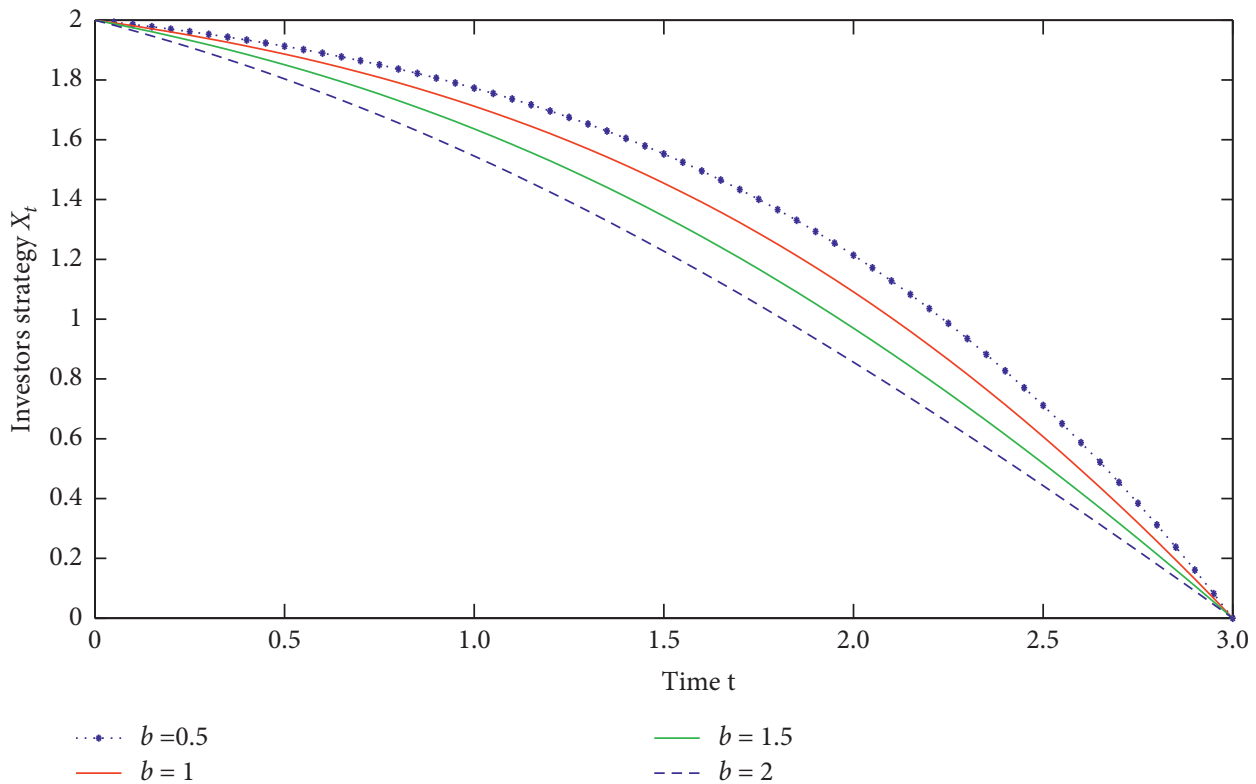

FIGURE 11: Influence of $b$ change on equation (34) when $\alpha=1, \rho=1$, and $x=2$.

Combined with the properties of Ito integral and equation (4), we obtain

$$
\text { minimize } \mathbb{E}[\mathscr{C}(X)]=\int_{0}^{T} e^{a+b\left(x-X_{t}\right)-\rho t} \dot{X}_{t}^{\alpha+1} \mathrm{~d} t .
$$

Using the Euler-Lagrange equation, the following nonlinear ODE with variable coefficients is obtained:

$$
2 \ddot{X}_{t}-2 \rho \dot{X}_{t}-b \dot{X}_{t}^{2}=0 .
$$

From [16], the solution of equation (43) is

$$
\int e^{-(b / \alpha+1) X_{t}} \mathrm{~d} X_{t}=C_{1} e^{(\rho / \alpha) t}+C_{2}
$$

Remark 6. In Theorem 6, we research the optimal liquidation behaviour when the market has exponential decay and temporary market impact and its coefficient are nonlinear functions. We can easily find that Theorem 4 is a special form when $\alpha=1$. 


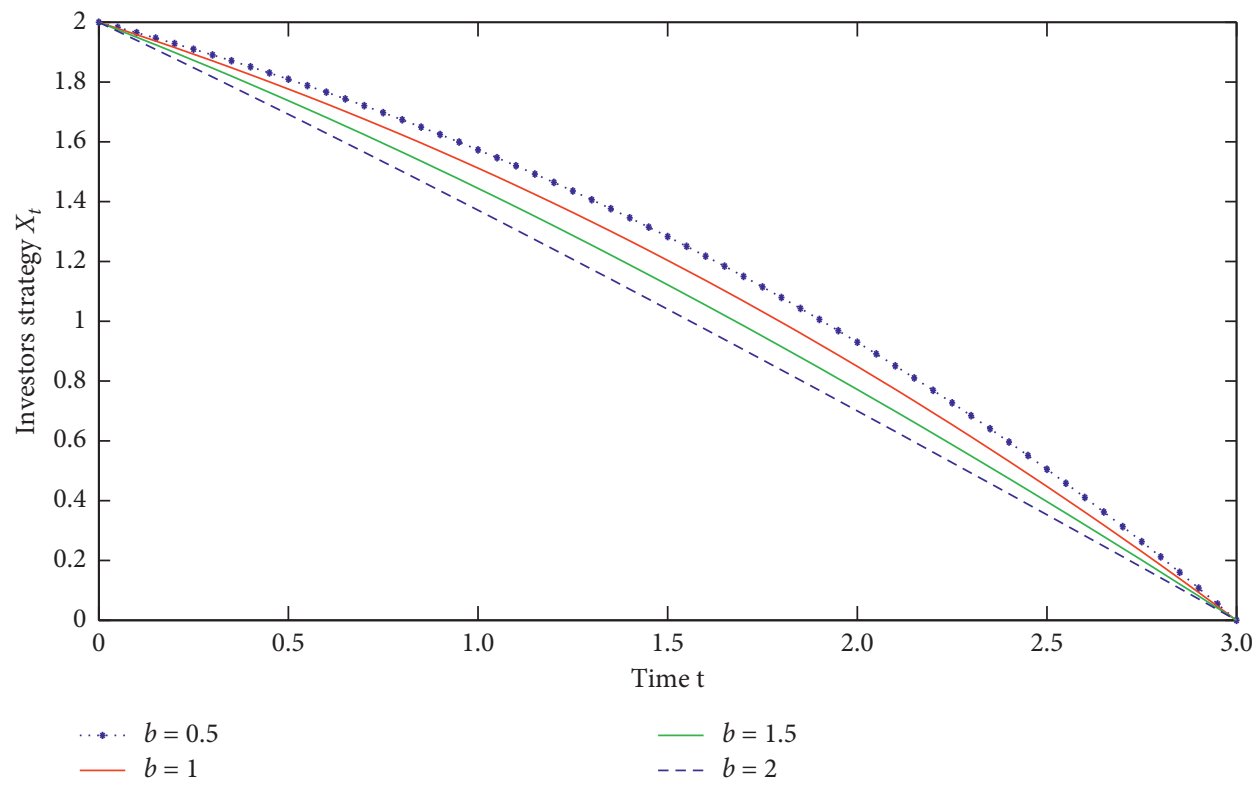

Figure 12: Influence of $b$ change on equation (44) when $\alpha=2, \rho=1$, and $x=2$.

\section{Numerical Simulation}

In Theorems 1, 3, 4, and 6, we give the specific form of the optimal investment behaviour for investors. Next, let $X_{0}=2, X_{t=3}=0, t \in[0,3]$, and we discuss the influence of parameters $\rho, a, b, x$, and $\alpha$ on the investor's optimal behaviour in four case, respectively.

From Figures 1-7, we find that investors realize that the risk of future trading is reduced when the market has decayed. Therefore, they reduce the amount $X_{t}$ and speed of liquidation in different situations and speed up the liquidation before the liquidation deadline. It can be found from Figure 1 that when the exponent of temporary impact item increases, investors gradually increase the speed and amount of liquidation in the early stage, reduce the uncertainty risk in the future, and obtain the maximized expected return. Through equations (14) and (26), we find that the optimal behaviour of investors is an increasing function of $a$. This is verified by Figures 2 and 3. Figures 2 and 3 discuss the change of investors' optimal liquidation behaviour with respect to the linear and nonlinear temporary impact, respectively. With the increase in $a$, investors reduce the speed of liquidation in the early stage so that they get the maximum return. At the same time, we also find that when $a$ is larger, the influence of $a$ will gradually decrease. Combined with equations (14) and (26) and Figures 4 and 5, we find that no matter whether the temporary market impact is linear or nonlinear, the optimal liquidation behaviour of investors is a decreasing function of $b$. With the increase in $b$, investors realize to reduce the liquidation speed in the early period so as to increase the return of future liquidation. Figures 6 and 7 discuss the situation of investors' liquidation behaviour with the change of $\rho$ under linear and nonlinear temporary impact, respectively. When investors find that $\rho$ is increasing, they realize that the holding risk caused by reducing the amount of $X_{t}$ in the early stage is reduced and increase the speed and amount of liquidation to maximize the return in the future. Therefore, when investors face these liquidation environments, they follow these liquidation strategies to minimize their execution cost in order to get the maximized returns.

Through equations (34) and (44), we see that the change of $a$ has no effect on the liquidation behaviour. From Figures 8-12, we further discuss the properties of the optimal liquidation behaviour about $b, \alpha$, and $\rho$. In Figure 8 , we find that when $a$ increases, investors realize that the risk of future trading will increase, and they will speed up the early liquidation to reduce the risk so as to obtain greater returns. From Figures 9 and 10, we find that when $\rho$ is larger, investors will reduce the speed and amount of $X_{t}$ in the early stage in order to reduce the risk of the exchange and get the maximized return. We study the influence of $b$ on $X_{t}$ under linear and nonlinear temporary impact in Figures 11 and 10. We find that when $b$ becomes larger, investors will accelerate the speed and amount of liquidation in the early stage and reduce the holding risk of assets, so as to maximize the returns.

From Figures 1 and 8, we find that the change of $\alpha$ has the same influence on the liquidation behaviour in two different situations. With the increase in $\alpha$, investors will speed up the early liquidation speed and amount so as to obtain the maximum returns. From Figures 4, 5, 11, and 12, the change of $b$ has the same effect on liquidation behaviour $X_{t}$. When $b$ gets larger, investors will liquidate faster so that they will reduce the risk of holding and get the maximum returns. Figures 6, 7, 4, and 10 investigate the relationship between decay factor $\rho$ and liquidation behaviour $X_{t}$. We find that the change of $\rho$ has the same effect on $X_{t}$ in four 
different situations. As $\rho$ gets larger, the investor will maximize the returns by reducing the number of trades made earlier.

\section{Conclusion}

In this paper, we extend the model of Cay and Muhle-Karbe [15] when there is an exponential decay factor in the market. When temporary market impact and its coefficient are linear and nonlinear, we obtain the optimal liquidation behaviour $X_{t}$ of investor which satisfies the second-order nonlinear ODE with variable coefficients. At the same time, we get specific forms of $X_{t}$ in four situations. Through the numerical simulation, the influence of parameter $\alpha, a, b$, and $\rho$ and initial holdings $x$ change on investors' liquidation strategy $X_{t}$ are shown. When the market environment meets these conditions, it has certain guiding significance for investors to liquidate assets in the future.

In this paper, we assume that the random factor is the standard Brownian motion and the coefficients are time consistent. In the future, we learn from the relevant research of Zhu [18] and study the optimal liquidation behaviour when the stochastic factor of the price impact model is G-Brownian motion and coefficients of model are time delay. At the same time, when the coefficients are unknown, we refer to the research of Zhu and Wang [19] and use the control method to further discuss the optimal liquidation behaviour for investors.

\section{Data Availability}

The data used to support the findings of this study are included within the article.

\section{Conflicts of Interest}

The authors declare that there are no conflicts of interest regarding the publication of this paper.

\section{Acknowledgments}

This work was supported by Key Research Base of Philosophy and Social Sciences for Colleges and Universities in Sichuan Province (KJJR2019-004), Talent Introduction Project of Xihua University (w202247), Fundamental Research Funds for the Central Universities (JBK2101036), and Scientific Research Project of Yibin University in 2019 (2019QD07).

\section{References}

[1] D. Bertsmas and A. Lo, "Optimal execution of execution costs," Journal of Finance Markets, vol. 1, pp. 1-50, 1998.

[2] R. Almgren and N. Chriss, "Optimal execution of portfolio transactions," Journal of Risk, vol. 3, no. 2, pp. 5-39, 2000.

[3] R. F. Almgren, "Optimal execution with nonlinear impact functions and trading-enhanced risk," Applied Mathematical Finance, vol. 10, no. 1, pp. 1-18, 2003.

[4] G. Huberman and W. Stanzl, "Price manipulation and quasiarbitrage,” Econometrica, vol. 72, no. 4, pp. 1247-1275, 2004.
[5] J. Gatheral and A. Schied, "Optimal trade execution under geometric brownian motion in the almgren and Chriss framework," International Journal of Theoretical and Applied Finance, vol. 14, no. 3, pp. 353-368, 2011.

[6] J. Gatheral, A. Schied, and A. Slynko, "Transient linear price impact and fredholm integral equations," Mathematical Finance, vol. 22, no. 3, pp. 445-3474, 2012.

[7] C.-A. Lehalle and E. Neuman, "Incorporating signals into optimal trading," Finance and Stochastics, vol. 23, no. 2, pp. 275-311, 2019.

[8] J. Gatheral, "No-dynamic-arbitrage and market impact," Quantitative Finance, vol. 10, no. 7, pp. 749-759, 2010.

[9] G. Curato, J. Gatheral, and F. Lillo, "Optimal execution with non-linear transient market impact," Quantitative Finance, vol. 17, no. 1, pp. 41-54, 2017.

[10] W. Barger and M. Lorig, "Optimal liquidation under stochastic price impact," International Journal of Theoretical and Applied Finance, vol. 21, no. 8, pp. 1850059-1850061, 2018.

[11] A. Bismuth, O. Guéant, and J. Pu, "Portfolio choice, portfolio liquidation, and portfolio transition under drift uncertainty," Mathematics and Financial Economics, vol. 13, no. 4, pp. 661-719, 2019.

[12] A. Schied and T. Zhang, "A state-constrained differential game arising in optimal portfolio liquidation," Mathematical Finance, vol. 27, no. 3, pp. 779-802, 2017.

[13] J. Vaes and R. Hauser, "Optimal execution strategy with an uncertain volume target," 2020, https://arxiv.org/pdf/1810. 11454.pdf.

[14] I. Ekren and S. Nadtochiy, "Utility-based pricing and hedging of contingent claims in almgren-chriss model with temporary price impact," 2020, https://arxiv.org/pdf/1910.01778.pdf.

[15] T. Cayé and J. Muhle-Karbe, "Liquidation with self-exciting price impact," Mathmatical Finance Economic, vol. 10, no. 17, pp. 15-28, 2016.

[16] D. P. Andrei and F. Z. Valentin, Handbook of Exact Solutions for Ordinary Differential Equations, Chapman Hall/CRC, Boca Raton, FL, USA, 2003.

[17] U. Horst and F. Naujokat, "Illiquidity and derivative valuation," 2018, https://arxiv.org/pdf/0901.0091.pdf.

[18] Q.X. Zhu, "Stabilization of stochastic nonlinear delay systems with exogenous disturbances and the event-triggered feedback control," IEEE Transactions on Automatic Control, vol. 64, no. 9, pp. 3764-3771, 2019.

[19] Q. Zhu and H. Wang, "Output feedback stabilization of stochastic feedforward systems with unknown control coefficients and unknown output function," Automatica, vol. 87, pp. 166-175, 2018. 